«Zeus demanda à ses serviteurs d'enchaîner Prométhée sur le plus haut sommet du mont Caucase où, chaque jour, pendant des siècles, un aigle vint ronger le foie sans cesse renaissant du malheureux. » Texte d'Eschyle dans Prométhée enchaîné $>$ La découverte de cellules souches au cœur du cerveau adulte bouleversa le dogme, central en neurobiologie, selon lequel le cerveau mature est une structure stable qui n'évolue plus. Depuis quelques années, les neurobiologistes constatent que, même parvenu au stade adulte, le cerveau reste encore capable de fabriquer des neurones qui s'intègrent aux réseaux existants. De façon constitutive, deux régions cérébrales (le bulbe olfactif et l'hippocampe) sont capables d'intégrer de nouveaux neurones et de tirer profit de cette jouvence neurale. Les nouveaux neurones sont issus de progéniteurs ayant des propriétés de cellules-souches neurales, et situés dans des régions particulières : la zone sous-ventriculaire pour les progéniteurs bulbaires, et la zone sous-granulaire pour les progéniteurs de l'hippocampe. Nombre de facteurs génétiques et épigénétiques régulant la prolifération des cellules souches, la migration, la différenciation et la survie des nouvelles cellules ont été identifiés et leurs mécanismes d'action élucidés. Cette capacité à produire de nouvelles cellules permet au cerveau adulte de s'adapter aux changements survenant dans son entourage; en cas de lésion ou de maladie, notamment, elle lui offre une possibilité de pouvoir se réparer. Dans cette synthèse, nous décrirons les différentes étapes par lesquelles une cellule-souche neurale produit des neurones destinés au bulbe olfactif, en insistant sur la façon dont cette production peut être régulée. Cette exploration nous conduira à décrire les résultats récents qui témoignent du potentiel réparateur des progéniteurs endogènes du système olfactif. Ces découvertes ouvrent la voie à de nouvelles stratégies visant à détourner, depuis leur zone germinative, les neurones nouvellement formés dans un cerveau adulte. <

\section{La neurogenèse bulbaire et son impact neurologique}

Antoine de Chevigny, Pierre-Marie Lledo

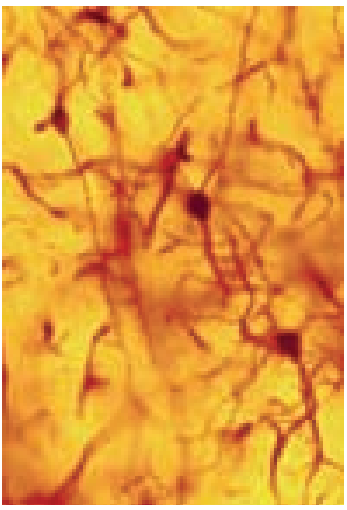

Institut Pasteur, Laboratoire Perception et mémoire, CNRS URA 2182, 25, rue du Docteur-Roux, 75724 Paris Cedex 15, France. pmlledo@pasteur.fr

\section{Neurogenèse secondaire du bulbe olfactif}

Jusque récemment, le cerveau adulte était considéré comme un organe dépourvu de toute capacité régénératrice et condamné à perdre inéluctablement ses éléments les plus précieux: les neurones. Pourtant, en 1969, Joseph Altman [1] observait déjà la production de nouvelles cellules chez le rat adulte, mais ces potentialités régénératrices suscitèrent un intérêt très limité. C'est dans les années 80 que la notion de production neurale chez l'adulte ${ }^{1}$ fut véritablement réhabilitée par les travaux portant sur le cerveau des canaris. II fallut encore attendre une décennie pour que les premières preuves du renouvellement des neurones fussent enfin favorablement accueillies chez les mammifères adultes.

On compte aujourd'hui deux structures principales capables de recevoir naturellement des nouveaux neurones. Chez les mammifères, il s'agit de l'hippocampe (structure limbique) et du bulbe olfactif (premier relais central de l'information olfactive). Dans les deux cas, les neurones nouvellement formés ont pour origine une zone germinative contenant des cellules souches: il s'agit de la zone sous-granulaire (ZSG) du gyrus denté de l'hippocampe et de la zone sous-ventriculaire (ZSV) bordant les ventricules cérébraux [2] (Figure 1). Cette neurogenèse constitutive est présente chez de nombreuses espèces, telles que les rongeurs [2], les prima-

${ }^{1}$ On parle de neurogenèse secondaire par opposition à celle qui participe à la construction du cerveau de l'embryon.

Article reçu le 23 novembre 2005, accepté le 4 janvier 2006. 
tes non humains $[3,4]$ et l'homme $[5,6]$. Néanmoins, de nombreux points distinguent ces deux structures: quantitativement, le nombre de nouveaux neurones bulbaires renouvelés chaque jour est au moins dix fois supérieur à celui de l'hippocampe; de plus, les jeunes neurones bulbaires nés dans la ZSV migrent sur de longues distances (environ $5 \mathrm{~mm}$ chez la souris) et à très grande vitesse (de l'ordre de $100 \mu \mathrm{m} / \mathrm{h}$ ), alors que la production neuronale dans l'hippocampe reste locale. S'il est certain que les régions cérébrales concernées par la neurogenèse secondaire sont associées à la mémoire et à l'apprentissage, la question de la signification physiologique de ce renouvellement reste posée.

On identifie trois types cellulaires dans la ZSV, sur des critères immunohistochimiques et ultrastructuraux [7]. Les neuroblastes (cellules de type A) forment des chaînes homotypiques et quittent la ZSV pour migrer en chaîne vers le bulbe olfactif, où ils se dif- férencient en interneurones locaux. Sont également présentes des cellules riches en protéines gliales fibrillaires (astrocytes nommés cellules de type $B$ ) et les cellules $C$, de forme sphérique, qui forment des amas épars apposés aux chaînes de neuroblastes. Des coupes sériées du courant de migration ont mis en évidence un agencement topographique particulier; les chaînes sont uniquement formées des corps cellulaires et des prolongements des neuroblastes, et les astrocytes, qui possèdent de multiples prolongements orientés selon la direction de migration, forment une gaine tubulaire enveloppant les neuroblastes: pendant la migration, les jeunes neurones se déplacent donc ensemble, les uns contre les autres, dans un tunnel formé par les cellules gliales. Les cellules en migration établissent des contacts

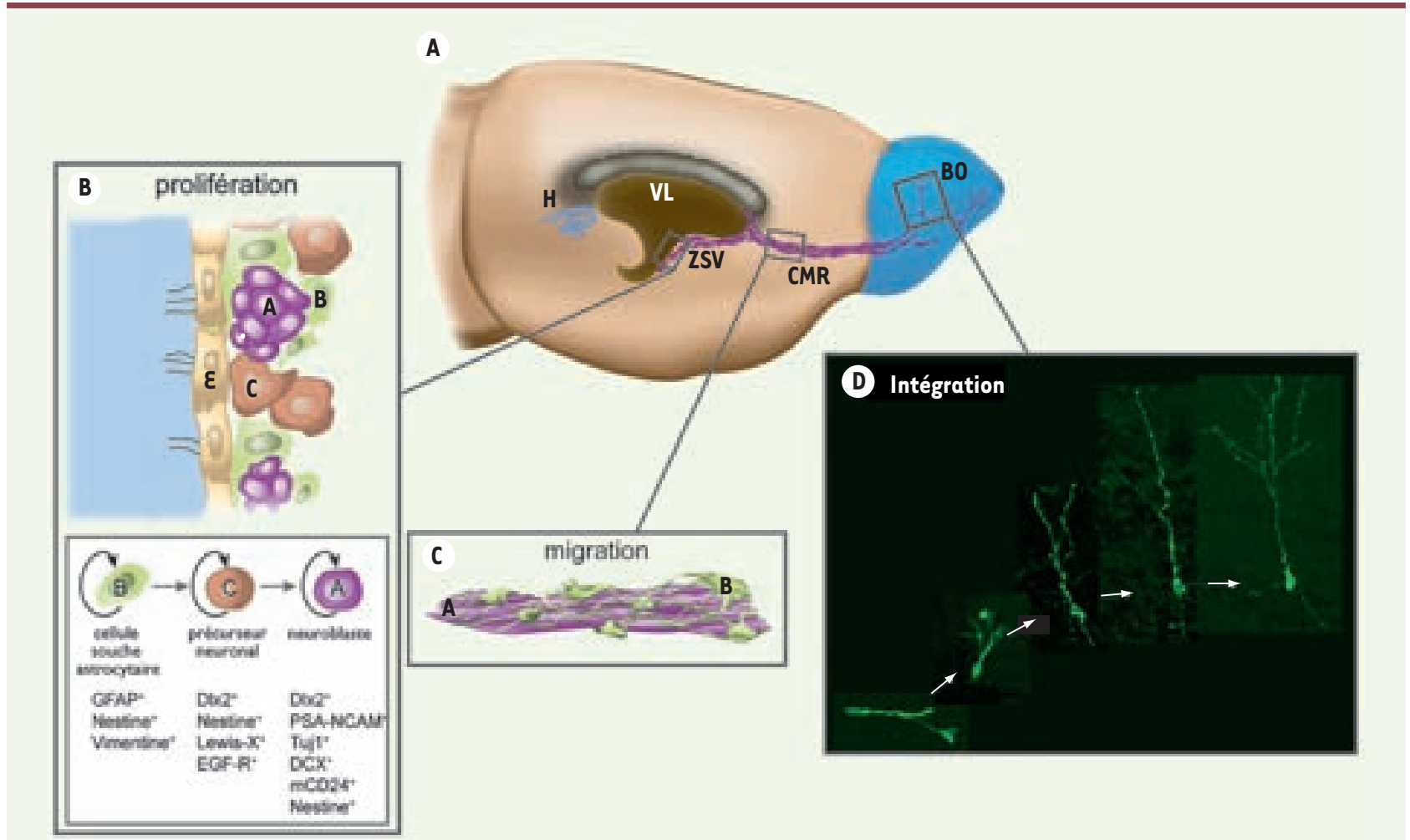

Figure 1. Étapes de la neurogenèse bulbaire chez le rongeur adulte. A. Coupe sagittale de cerveau de rongeur adulte où figurent les précurseurs des neurones bulbaires (cellules violettes). Ces neuroblastes, produits pour la plupart dans la zone sous-ventriculaire (ZSV), migrent au sein du courant de migration rostrale (CMR) en direction du bulbe olfactif (BO), où ils se différencient en interneurones bulbaires (violets). La ZSV borde la paroi latérale des ventricules latéraux (VL). H : hippocampe. B. Section frontale de cerveau de souris adulte indiquant la composition cellulaire et l'architecture de la ZSV. Chez l'adulte, la monocouche des cellules épendymaires $(\varepsilon)$ isole le ventricule latéral (en bleu) des autres cellules de la ZSV. Les cellules souches astrocytaires (B) sont capables de s'autorenouveler et d'engendrer des progéniteurs neuronaux à division rapide, les cellules de type $C$. Celles-ci produisent à leur tour des neuroblastes (cellules $A$ ), qui forment des chaînes parallèles aux VL. Les marqueurs spécifiques des cellules $B, C$ et $A$ sont présentés dans l'encadré du bas. $C$. Les chaînes de neuroblastes migrent tangentiellement vers le bulbe olfactif, le long d'un tunnel formé par les cellules astrocytaires; ce tunnel est le courant de migration rostrale. $D$. Dans la région bulbaire du CMR, les neuroblastes (marqués ici par la GFP) s'individualisent et s'orientent radiairement pour atteindre leurs positions finales dans le bulbe olfactif et se différencier en interneurones locaux. GFP : green fluorescent protein; GFAP : glial fibrillary acidic protein; Dix2 : Distalless-related homeobox 2 ; عGF-R : epidermal growth factor-receptor; PSA-NCAM : polysialylated neural cell adhesion molecule; Tuj : neuron-specific tubulin-1; DCX : doublecortin. 


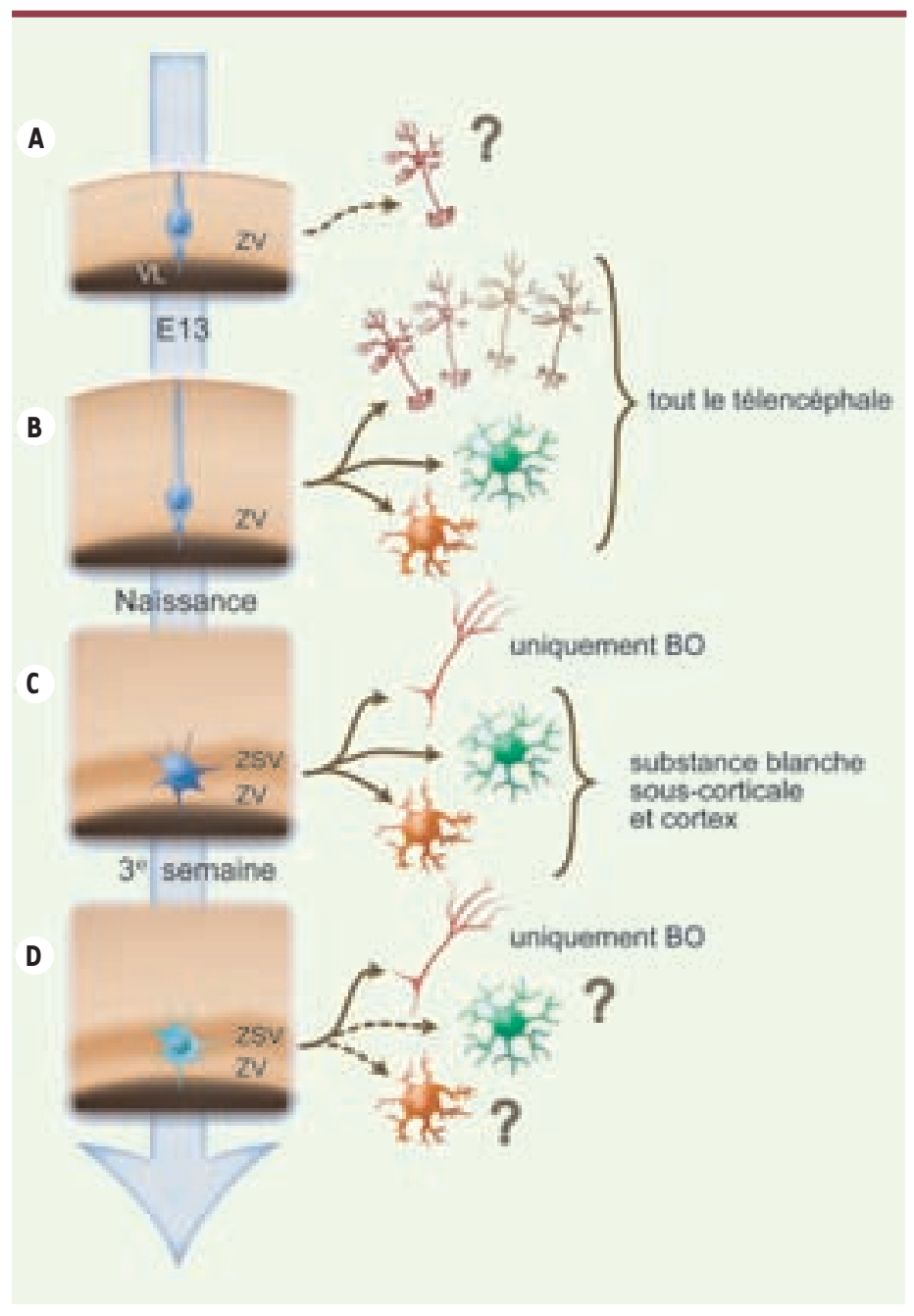

Figure 2. Lignage des progéniteurs neuraux endogènes au cours du développement télencéphalique du rongeur. A. Les premières cellules constituant le tube neural, à l'origine du système nerveux central, sont les cellules neuroépithéliales. Elles ont un corps cellulaire apposé à la paroi ventriculaire, un cil unique en contact avec le ventricule, et un prolongement apical court qui projette jusqu'à la surface piale. Ces cellules seraient les premières progénitrices durant le développement, car elles produiraient des neurones lors de divisions asymétriques. $\boldsymbol{B}$. Au stade $\varepsilon 13$, les cellules neuro-épithéliales évoluent en cellules de glie radiaire, qui persistent jusqu'à la naissance. Leur prolongement apical est plus long car l'épaisseur du cerveau a augmenté. Ces cellules sont les progéniteurs de la plupart des neurones télencéphaliques, mais produisent aussi des astrocytes et des oligodendrocytes : elles sont donc multipotentes. C. À la naissance, la plupart des cellules de la glie radiaire rétractent leurs prolongements apicaux. Elles se différencient en cellules astrocytaires, qui demeurent multipotentes durant les deux premières semaines de la vie. Les neurones engendrés par ces cellules sont destinés au bulbe olfactif (BO), alors que les cellules gliales qu'elles produisent sont destinées au cortex. D. Certains de ces progéniteurs neuraux ayant des caractéristiques astrocytaires persistent chez l'adulte. Néanmoins, leur potentiel de différenciation in vivo se réduit, puisqu'elles ne produiraient que des neurones bulbaires. très étroits entre elles, mais jamais avec les cellules gliales environnantes. Autre particularité, les neuroblastes possèdent la capacité de se diviser durant leur déplacement, alors même que certaines protéines caractéristiques des neurones sont déjà présentes.

Si l'on élimine sélectivement les cellules $A$ et $C$ à l'aide d'antimitotiques, les cellules $B$ peuvent régénérer d'abord la population de cellules $C$, puis celle des cellules $A[8]$. Ce travail permet de montrer les relations hiérarchiques du lignage cellulaire : les cellules $B$ à division très lente (les cellules souches neurales) donnent naissance aux cellules $C$ à division rapide (progéniteurs neuraux), qui produisent à leur tour les neuroblastes (type $A$ ). Les cellules $B$ sont issues de la glie radiaire striatale, elle-même issue de cellules neuro-épithéliales (Figure 2).

Une fois le bulbe olfactif atteint, les cellules $A$ s'individualisent, migrent perpendiculairement à la surface cérébrale puis se différencient en interneurones granulaires ou périglomérulaires. Les interneurones granulaires sont tous de nature GABAergiques. En revanche, la population d'interneurones périglomérulaires est très hétérogène: ils peuvent être GABAergiques et dopaminergiques à la fois, ou seulement GABAergiques [9]. Enfin, l'approvisionnement bulbaire en nouveaux neurones dépend de l'intensité de l'activité sensorielle: le degré d'activité bulbaire influence directement le nombre de nouveaux neurones qui, après avoir atteint le bulbe olfactif, pourront survivre plus ou moins longtemps $[10,11]$. Cette propriété permettrait d'ajuster le traitement de l'information aux variations d'activité des afférences sensorielles.

\section{Contrôle de la neurogenèse secondaire : le cas du bulbe olfactif}

Nombre de facteurs impliqués dans la régulation de la neurogenèse bulbaire ont été identifiés, améliorant ainsi la compréhension des mécanismes de prolifération cellulaire, de migration, de spécification phénotypique et de survie cellulaire.

Des facteurs de croissance, comme l'EGF (epidermal growth factor) et le FGF (fibroblast growth factor), stimulent globalement la prolifération cellulaire des progéniteurs neuraux et ont des effets variables sur la spécification neurale [12]. Dans la ZSV adulte, la spécification neuronale est ultramajoritaire par rapport à la spécification gliale; cette situation résulterait du blocage de l'activation des récepteurs des protéines 
morphogéniques osseuses (BMP) par le facteur Noggin [13]. Deux facteurs de transcription, Pax6 et Olig2, favorisent la spécification neuronale et gliale, respectivement [14]. Chez la souris adulte, l'effet proneuronal intrinsèque de Pax6 est primordial, car le blocage de son activité endogène dans la ZSV empêche la formation des neuroblastes et, par conséquent, l'arrivée de nouveaux neurones dans le circuit bulbaire. Depuis la ZSV, la migration tangentielle est contrôlée par deux facteurs libérés par le septum, Slitl et 2, qui repoussent les neuroblastes vers la partie rostrale du cerveau [15]. Notons aussi que le bulbe olfactif synthétise des facteurs attractifs qui faciliteraient la migration des cellules nouvellement produites $[16,17]$. La migration radiaire est quant à elle contrôlée par l'action de protéines extracellulaires : la reeline [18], la ténascine-R (TN-R) [19] et la prokinéticine [20], toutes produites par le bulbe olfactif. Fait notable, les neuroblastes

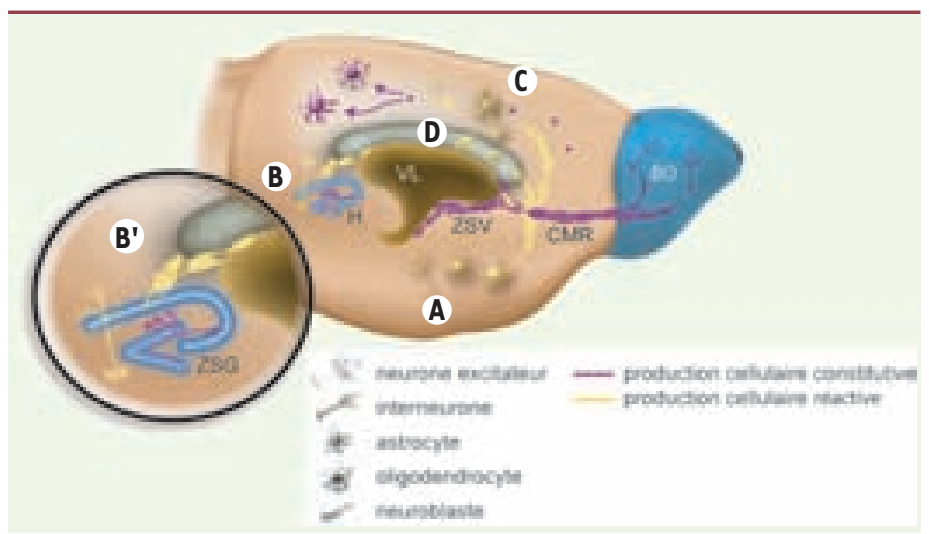

Figure 3. Neurogenèses constitutive et réactive. Les progéniteurs neuraux endogènes du télencéphale sont représentés en violet. Ils sont localisés dans la zone sous-ventriculaire (ZSV) et le courant de migration rostrale (CMR) pour le système olfactif, et dans la zone sous-granulaire (ZSG) du gyrus dentelé pour l'hippocampe (H). Les progéniteurs de la ZSV et du CMR se différencient en interneurones granulaires et périglomérulaires dans le bulbe olfactif (BO), alors que ceux de la ZSG se différencient en neurones de projection granulaires dans le gyrus dentelé (en violet). En jaune sont représentés les progéniteurs neuraux recrutés par des zones ectopiques dans des modèles animaux de maladies ou de lésions neurodégénératives. A. Dans un modèle d'ischémie focale affectant principalement le striatum, des progéniteurs de la ZSV sont recrutés et certains d'entre eux se différencient de manière appropriée en neurones épineux de taille moyenne [3]. B-B'. Dans un autre modèle d'ischémie touchant spécifiquement la couche CAl de l'hippocampe, des progéniteurs de la région caudale de la ZSV sont attirés vers la zone lésée, où ils remplacent des neurones morts. Ce taux de remplacement neuronal est augmenté après une instillation intraventriculaire de facteurs de croissance comme l'EGF (epidermal growth factor) et le FGF (fibroblast growth factor), responsable de l'amélioration fonctionnelle des animaux [28]. C. Après destruction spécifique de neurones corticothalamiques ou corticospinaux par photolyse, certains d'entre eux sont remplacés par des progéniteurs issus de la ZSV [22, 23]. D. Dans un modèle de maladie autoimmune démyélinisante (mimant la sclérose en plaque), des précurseurs du CMR migrent vers des zones de substance blanche démyélinisée et se différencient en oligodendrocytes et en astrocytes [30]. de la ZSV et du courant de migration sont recrutés par la TN-R placée en situation ectopique. Ainsi, de nouveaux neurones peuvent être acheminés vers le striatum lorsqu'il lui est permis de sécréter la TN-R [19]. Enfin, une fois intégrés, les nouveaux neurones ont une survie qui dépend étroitement des stimuli olfactifs : le rôle de l'activité sensorielle sur la survie cellulaire se ferait, au moins en partie, par l'intermédiaire du glutamate libéré par les neurones de projection [21].

\section{Vers une utilisation des cellules souches neurales en clinique?}

Si le renouvellement neuronal à partir des progéniteurs de la ZSV adulte se restreint au bulbe olfactif dans des conditions normales, il peut concerner d'autres régions dans certaines conditions pathologiques (Figure 3). Ces régions non neurogéniques peuvent accueillir de nouveaux neurones lors d'une maladie neurodégénérative ou d'un trauma, ou après un accident vasculaire. Différents modèles lésionnels offrent des conditions favorables pour étudier cette neurogenèse réactive.

L'équipe du Dr Macklis a mis au point une technique de photolyse permettant d'éliminer sélectivement des neurones corticaux par le déclenchement d'une apoptose massive : dans ce modèle, ils ont mis en évidence la production de nouveaux neurones corticothalamiques [22] puis, ultérieurement, de motoneurones corticospinaux [23], capables de survivre plusieurs mois et de contacter les régions contralatérales appropriées. La majorité de ces nouveaux neurones semblent issus de la ZSV. Si ces résultats sont accueillis avec optimisme, il n'en demeure pas moins que ce modèle lésionnel souffre de certaines limites: les lésions utilisées au cours de ces expériences n'atteignent que les neurones de projection, sans affecter les cellules gliales et autres interneurones: par ailleurs, le signal de mort cellulaire est donné de façon artificiellement synchronisée, entraînant une situation cliniquement peu pertinente. Une autre étude montre que la production de neurones striataux est possible après l'induction d'une ischémie transitoire chez le rongeur [24]; de nouveau, c'est la ZSV qui semble produire les neurones désirés. II semble donc que le cerveau mature soit bien capable de remplacer certaines catégories neuronales lorsque la mort cellulaire est massive. Le remplacement des neurones se ferait essentiellement par recrutement des progéniteurs de la ZSV, grâce aux modifications micro-environnementales produites par la lésion [25].

Si l'espoir levé par ces résultats est grand, un obstacle majeur reste néanmoins présent: la proportion de nouveaux neurones produits est extrêmement faible 
par rapport au nombre important de neurones disparus [22-24]. Concernant l'ischémie, non seulement une grande partie des progéniteurs de la ZSV recrutés par le striatum se différencient en astrocytes, mais la majorité des néo-neurones striataux engendrés est rapidement éliminée [24]. II est donc indispensable que des stratégies qui visent à contrôler la prolifération, le recrutement, la spécification et la survie cellulaires soient envisagées de façon concertée, afin que la récupération fonctionnelle puisse être significative. Dans cette optique, trois facteurs de croissance, l'EGF, le FGF et le TGF (transforming growth factor) ont été testés, et des résultats saisissants obtenus. La lésion des afférences dopaminergiques striatales provenant de la substance noire reproduit chez la souris les signes cliniques caractéristiques de la maladie de Parkinson. Si l'on injecte le TGF, un ligand endogène du récepteur de l'EGF, dans le striatum, une nette amélioration du comportement locomoteur est observée [26]. Si l'effet prolifératif et attractif des facteurs de croissance sur les progéniteurs de la ZSV était attendu [12], la différenciation de ces progéniteurs en neurones dopaminergiques reste en revanche fort surprenante, puisque le striatum n'héberge aucun neurone dopaminergique dans des conditions physiologiques normales. D'ailleurs, en tentant de reproduire ces résultats, l'équipe du

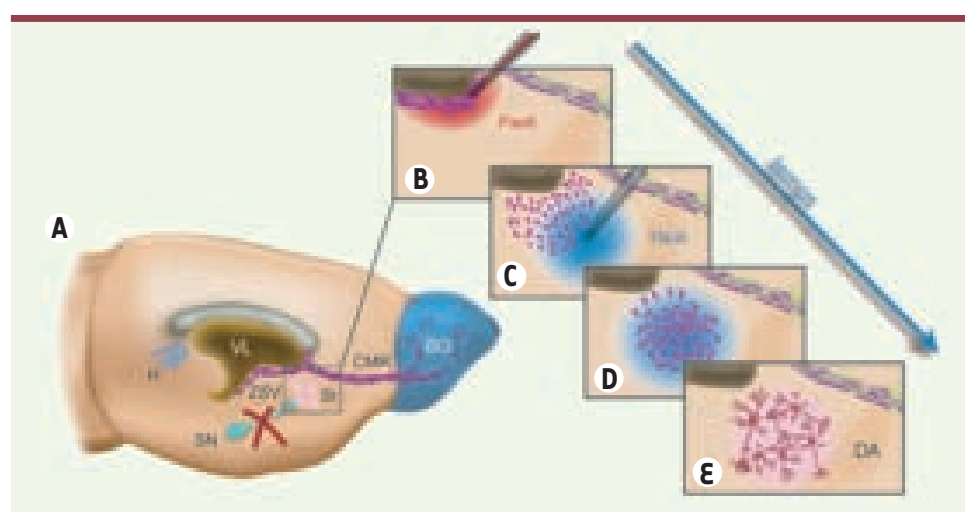

Figure 4. Stratégie de traitement d'un modèle murin de maladie de Parkinson. Ce modèle repose sur le recrutement de progéniteurs endogènes dont le destin est préalablement sélectionné. $A$. Coupe sagittale de cerveau de souris. Les neurones dopaminergiques de la substance noire (SN, en vert), libérant la dopamine (DA, rose) dans le striatum, sont lésés après l'injection de 6-hydroxydopamine (croix rouge). La baisse de la libération de dopamine dans le striatum entraîne une altération des comportements locomoteurs, ce qui contitue un modèle murin de la maladie de Parkinson. B. Nous proposons d'instiller dans la ZSV un vecteur viral contenant le gène codant pour la protéine Pax6 (en rouge): les neuroblastes ainsi transduits sont orientés vers un destin dopaminergique [14]. C, D. Une instillation striatale de ténascine- $R$ purifiée (TN-R, en bleu) recruterait alors ces neuroblastes [19]. $\varepsilon$. Une fois un grand nombre de neuroblastes attirés dans la zone désafférentée, la teneur en dopamine pourrait atteindre des valeurs initiales responsables d'une amélioration fonctionnelle $(\varepsilon)$. BO : bulbe olfactif; CMR : courant de migration rostrale; VL : ventricule latéral; ZSV : zone sous-ventriculaire. piques [28].
Dr Isacson a confirmé l'effet prolifératif et le recrutement de progéniteurs de la ZSV dans le striatum, mais n'a pas observé de nouveaux neurones dopaminergiques [27]. II est donc nécessaire, avant d'entériner l'une ou l'autre version du phénomène, d'en obtenir avant tout confirmation. fération des progéniteurs endogènes et la régénération de plus de $40 \%$ des neurones pyramidaux sont observées chez les animaux ayant reçu les deux facteurs de croissance. Dans ce cas, l'origine des nouveaux neurones semble être la partie caudale de la ZSV. Fait notable, ces chercheurs démontrent que les néo-neurones pyramidaux survivent au moins six mois après l'accident vasculaire, et notent une amélioration importante des fonctions hippocamnodules hyperplasiques sur les parois ventriculaires [27]. II est donc indispensable d'approfondir nos connaissances sur les facteurs endogènes régulant la neurogenèse adulte, afin d'élaborer des stratégies reposant sur des techniques de réparation mieux contrôlées, plus efficaces et dépourvues d'effets secondaires. Dans ce contexte, les récentes découvertes de notre laboratoire fournissent des informations substantielles. L'expression ectopique, dans le parenchyme striatal, de TN-R, un facteur essentiel à la migration radiaire au sein du bulbe, est suffisante pour recruter les neuroblastes de la ZSV [19]. Par ailleurs, nous avons récemment identifié un déterminant majeur de la spécification dopaminergique des progéniteurs de la ZSV : le facteur de transcription Pax6 [14]. Une fois dans le bulbe olfactif, seuls les progéniteurs exprimant Pax6 se différencient en neurones dopaminergiques et le maintien forcé de l'expression de Pax6 dans les neuroblastes du courant de migration permet une production massive de neurones dopaminergiques. Ce travail met donc en évidence l'un des mécanismes moléculaires par lesquels le destin des nouveaux neurones peut être préalablement choisi. La Figure 4 propose une stratégie de traitement de la maladie de Parkinson, qui repose sur l'injection de vecteurs viraux permettant l'expression de Pax6 dans la ZSV, afin de spécifier un destin dopaminergique aux neurones, suivie de l'instillation striatale de TN-R pour recruter les progéniteurs ainsi prédifférenciés.

\section{Conclusions}

Un pas important vers l'utilisation des cellules souches neurales endogènes vient d'être théoriquement franchi. Les résultats récents ouvrent d'intéressantes perspectives pour 
le développement de thérapies cellulaires ayant pour objectif de réparer le cerveau. De nouveaux neurones pourraient être recrutés à partir de progéniteurs nés dans la ZSV (par instillation de facteurs attractifs), et leur destin cellulaire choisi à l'aide de vecteurs viraux garantissant l'expression à long terme des facteurs de transcription appropriés. À partir de modèles murins et primates, il reste à contrôler la survie des néo-neurones ainsi déviés de leur chemin naturel, à vérifier leurs propriétés fonctionnelles et à démontrer qu'ils s'intègrent de façon appropriée.

Néanmoins, avant d'envisager tout bénéfice clinique pour cette neurogenèse secondaire, il est important de prendre en compte l'étiologie de la perte neuronale. La localisation du territoire lésé, notamment, influe de manière importante sur le remplacement neuronal : si cette lésion est trop éloignée de la ZSV, le remplacement semble ainsi impossible [24]. La dimension temporelle constitue elle aussi un facteur limitant: l'induction rapide d'une mort cellulaire, lors d'un accident vasculaire, par exemple, permet un recrutement plus efficace des neuroblastes que celle observée dans les maladies neurodégénératives, dont l'instauration est souvent lente. Une mort cellulaire massive et rapide semble donc plus efficace pour altérer les signaux micro-environnementaux exerçant une fonction répressive sur l'acheminement et le recrutement des nouveaux neurones ou, à l'inverse, pour libérer en quantité substantielle les signaux favorables au recrutement des progéniteurs endogènes. Enfin, le facteur génétique de certaines maladies dégénératives peut également représenter un obstacle majeur : les nouveaux neurones produits à partir de progéniteurs endogènes portant en effet la même anomalie génétique, survivront-ils une fois acheminés dans les tissus lésés?

La régénération par remplacement cellulaire à partir des progéniteurs endogènes (dont le patrimoine génétique peut être modifié par des vecteurs viraux) en est à ses débuts. On ne sait pas encore si les avancées réalisées sur les modèles animaux seront directement transposables à l'homme ${ }^{2}$. Pourtant, il est certain que cette approche présente des avantages réels par rapport aux stratégies thérapeutiques fondées sur l'emploi de cellules souches embryonnaires ou fœtales: elle évite notamment les problèmes éthiques, de rejet immunologique par l'hôte et de mutations génétiques qui peuvent apparaître durant le maintien en culture des cellules souches [29]. La découverte des facteurs endogènes régulant la neurogenèse secondaire devrait certainement permettre d'améliorer substantiellement le recrutement, la survie et la différenciation des progéniteurs neuraux.

Gageons que le supplice de Prométhée, enchaîné pour longtemps à son rocher, puisse nous éclairer un peu plus sur nos réels pouvoirs régénérateurs, sans attendre qu'Héraklès vienne nous libérer ! $\diamond$

\section{SUMMARY}

Olfactory bulb neurogenesis and its neurological impact

Contrary to the long-held dogma according to which the adult mammalian brain does not produce neurons anymore, neuronal turnover has been reported in two discrete areas of the adult brain: the hippocampus and the olfactory bulb. Adult-generated neurons are produced from neural stem cells located

${ }^{2}$ Bien que la neurogenèse secondaire soit présente dans l'hippocampe humain [5], et que l'on puisse isoler des cellules souches à partir de la ZSV humaine [6], la démonstration d'une production neuronale in vivo par cette dernière région n'a pas encore été faite. in the hippocampal subgranular zone and the subventricular zone of the lateral ventricles. Recently, number of genetic and epigenetic factors that modulate proliferation of stem cells, migration, differentiation and survival of newborn neurons have been characterized. We know that neurogenesis increases in the diseased brain, after stroke or after traumatic brain injury. Importantly, progenitors from the subventricular zone, but not from the subgranular zone, are incorporated at the sites of injury, where they replace some of the degenerated neurons. Thus, the central nervous system has the capacity to regenerate itself after injury and, today, researchers develop strategies aimed at promoting neurogenesis in diseased areas. This basic research is attracting a lot of attention because of the hope that it will lead to regeneration and reconstruction therapy for the damaged brain. In this review, we discuss major findings concerning the organization of the neurogenic niche located in the subventricular zone and examine both intrinsic and extrinsic factors that regulate adult neurogenesis. Then, we present evidences for the intrinsic capability of the adult brain for cell replacement, and shed light on recent works demonstrating that one can greatly enhance appropriate brain cell replacement by using molecular cues known to endogenously control proliferation, migration, differentiation and/or survival of subventricular zone progenitors. Finally, we review some of the advantages and limits of strategies aimed at using endogenous progenitors and their relevance to human clinics. $\diamond$

\section{REMERCIEMENTS}

Nous remercions chaleureusement Cécile Galliot, pour sa production iconographique, et Jacques Epelbaum, pour ses commentaires bienveillants concernant notre manuscrit. Notre recherche est soutenue par l'aide financière du consortium Inserm/Ministère délégué Recherche et Nouvelles Technologies/AFM/JDRFI (Appel d'Offres 2003 «Cellules Souches Adultes »), l'Agence nationale de la recherche (ANR-05-NEUR-028), la Fédération pour la recherche sur le cerveau (Appel d'Offres 2004), l'Institut Pasteur (GPH nº 7 , «Stem cells») et le CNRS (DRI «Cellules Souches »).

\section{RÉFÉRENCES}

1. Altman J. Autoradiographic and histological studies of postnatal neurogenesis. IV. Cell proliferation and migration in the anterior forebrain, with special reference to persisting neurogenesis in the olfactory bulb. J Comp Neurol $1969 ; 137: 433-58$.

2. Lois $C$, Alvarez-Buylla A. Long-distance neuronal migration in the adult mammalian brain. Science $1994 ; 264$ : 1145-8.

3. Kornack DR, Rakic P. The generation, migration, and differentiation of olfactory neurons in the adult primate brain. Proc Natl Acad Sci USA 2001 ; $98: 4752-7$.

4. Pencea V, Bingaman KD, Freedman LJ, Luskin MB. Neurogenesis in the subventricular zone and rostral migratory stream of the neonatal and adult primate forebrain. Exp Neurol 2001; 172: 1-16.

5. Eriksson PS, Perfilieva E, Bjork-Eriksson T, et al. Neurogenesis in the adult human hippocampus. Nat Med $1998 ; 11: 1313-7$.

6. Sanai N, Tramontin AD, Quinones-Hinojosa A, et al. Unique astrocyte ribbon in adult human brain contains neural stem cells but lacks chain migration. Nature $2004 ; 427: 740-4$. 
7. Doetsch F, Garcia-Verdugo JM, Alvarez-Buylla A. Cellular composition and three-dimensional organization of the subventricular germinal zone in the adult mammalian brain. J Neurosci $1997 ; 17: 5046-61$.

8. Doetsch F, Caille I, Lim DA, et al. Subventricular zone astrocytes are neural stem cells in the adult mammalian brain. Cell $1999 ; 97: 703-16$.

9. Halasz N, Shepherd GM. Neurochemistry of the vertebrate olfactory bulb. Neuroscience 1983 ; $10: 579-619$

10. Rochefort C, Gheusi G, Vincent JD, Lledo PM. Enriched odor exposure increases the number of newborn neurons in the adult olfactory bulb and improves odor memory. J Neurosci 2002 ; 22 : 2679-89.

11. Saghatelyan A, Roux P, Migliore M, et al. Activity-dependent adjustments of the inhibitory network in the olfactory bulb following early postnatal deprivation. Neuron 2005 ; 46: 103-16.

12. Gage FH. Mammalian neural stem cells. Science $2000 ; 287: 1433-8$.

13. Lim DA, Tramontin AD, Trevejo JM, et al. Noggin antagonizes BMP signaling to create a niche for adult neurogenesis. Neuron $2000 ; 28: 713-26$.

14. Hack MA, Saghatelyan A, de Chevigny A, et al. Neuronal fate determinants of adult olfactory bulb neurogenesis. Nat Neurosci $2005 ; 8: 865-72$.

15. Lie DC, Song H, Colamarino SA, et al. Neurogenesis in the adult brain: new strategies for central nervous system diseases. Annu Rev Pharmacol Toxicol 2004 ; 44 : 399-421.

16. Murase S, Horwitz AF. Deleted in colorectal carcinoma and differentially expressed integrins mediate the directional migration of neural precursors in the rostral migratory stream. J Neurosci $2002 ; 22: 3568-79$.

17. Liu G, Rao Y. Neuronal migration from the forebrain to the olfactory bulb requires a new attractant persistent in the olfactory bulb. J Neurosci $2003 ; 23: 6651-9$.

18. Hack I, Bancila M, Loulier K, et al. Reelin is a detachment signal in tangential chainmigration during postnatal neurogenesis. Nat Neurosci $2002 ; 5$ : 939-45.

19. Saghatelyan A, de Chevigny A, Schachner M, Lledo PM. Tenascin-R mediates activitydependent recruitment of neuroblasts in the adultmouse forebrain. Nat Neurosci 2004 ; $7: 347-56$.

20. Ng KL, Li JD, Cheng My, et al. Dependence of olfactory bulb neurogenesis on prokineticin 2 signaling. Science $2005 ; 308$ : $1923-7$.

21. Fiske BK, Brunjes PC. NMDA receptor regulation of cell death in the rat olfactory bulb. J Neurobiol $2001 ; 47: 223-32$.
22. Magavi SS, Leavitt BR, Macklis JD. Induction of neurogenesis in the neocortex of adult mice. Nature 2000 ; 405 : 951-5.

23. Chen J, Magavi SS, Macklis JD. Neurogenesis of corticospinal motor neurons extending spinal projections in adult mice. Proc Natl Acad Sci USA 2004 ; 101 : 16357-62.

24. Arvidsson A, Collin T, Kirik D, et al. Neuronal replacement from endogenous precursors in the adult brain after stroke. Nat Med 2002 ; $8: 963-70$.

25. Jin K, Mao XO, Sun Y, et al. Stem cell factor stimulates neurogenesis in vitro and in vivo. J Clin Invest $2002 ; 110: 311-9$.

26. Fallon J, Reid $S$, Kinyamu $R$, et al. In vivo induction of massive proliferation, directed migration, and differentiation of neural cells in the adult mammalian brain. Proc Natl Acad Sci USA 2000 ; 97 : 14686-91.

27. Cooper 0 , Isacson 0 . Intrastriatal transforming growth factor alpha delivery to a model of Parkinson's disease induces proliferation and migration of endogenous adult neural progenitor cells without differentiation into dopaminergic neurons. J Neurosci 2004 ; $24: 8924-31$

28. Nakatomi H, Kuriu T, Okabe S, et al. Regeneration of hippocampal pyramidal neurons after ischemic brain injury by recruitment of endogenous neural progenitors. Cell $2002 ; 110: 429-41$.

29. Mitchell BD, Emsley JG, Magavi SS, et al. Constitutive and induced neurogenesis in the adult mammalian brain: manipulation of endogenous precursors toward CNS repair. Dev Neurosci 2004 ; 26 : 101-17.

30. Picard-Riera N, Nait-Oumesmar B, Baron-Van Evercooren A. Endogenous adult neural stem cells : limits and potential to repair the injured central nervous system. J Neurosci Res $2004 ; 76: 223-31$.

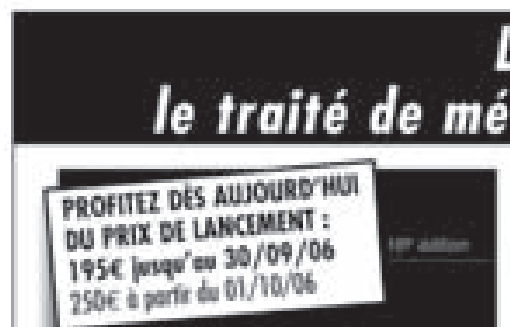

HARRISON

Médecine

Interne

0.650

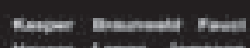

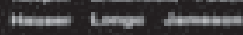

\section{LA $16^{\circ}$ EDITION DU HARRISON,}

TIRÉS À PART

P.M. Lledo
A paralore Sepoentive $2006-2890$ puges et 1300 alustrations en NB ef en couleur

\section{- pour la première fois en quadrichromie : HARRISON : Principes de Médecine Interne}

\author{
Kasper, Braunwald, Faud, Hauser, Longo, Jameson.
}

Nouvelle édition d'un ouvrage de référence incontoumable, traduit par des médecins specialistes dans chaque discipline dont une première partie traite de la pratique médicale, suivie de 15 parties consacrées aux symptómes, syndromes et maladies dans toutes les disciplines médicales

Au total $\bullet$ un ouvrage exhaustif de 2880 pages et 1300 illustrations en noir et blanc et en couleurs $\bullet \mathbf{2 0}$ nouveaux chapitres consacres notamment à la prevention, aux malades en fin de vie, a la périmenopause, a l'angor instable, a Finsuffisance respiratoire aiguê, au SRAS, aux nouveaux traitements de Thépatite virale, aux stroke-centers, etc. $\bullet$ des encadrés récapitulatifs de prise en charge des patients en fin de chapitre, de très nombreux algorithmes, tableaux et figures, des tableaux sur les sites internet proposant les donnees les plus détaillees sur certains traitements, etc.

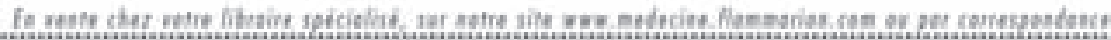
EON DE COMMANOE a ietournet a

FAMMLaOoN MEDTCINF - 87, Quai Panhard et Levassor - 75647 papus Cedex 13

NOM Frenom Vie

Code posta

le commande et je itgle of pient par cheque bancaire a rontre de flammarion fune facture acoultite ara joines au colisy

I PRINCPES DE MEDECWE INTERNE - HARRISON - 16 edition $1+5$ E de participation aur fras de porti jusqu'au 30 io9/05 7 PRINCIPES DE MEDECNE INTERNE - HARRISON - 16 edition + t 5 E de participation aur fras de porti a partir du $01 / 10 / 06$ 